\title{
Hyphal Morphology and Elongation Alterations in Aspergillus nidulans Provoked by the Diterpene Kaurenoic Acid
}

\author{
Janice A. Rafael ${ }^{1,2 *}$, Nilton S. Arakawa ${ }^{3}$, Sérgio R. Ambrosio ${ }^{4}$, \\ Fernando B. Da Costa ${ }^{1}$, Suraia Said ${ }^{1}$ \\ ${ }^{1}$ Faculdade de Ciências Farmacêuticas de Ribeirão Preto, Universidade de São Paulo, Ribeirão Preto, Brazil \\ ${ }^{2}$ Centro de Ciências da Saúde, Universidade do Vale do Paraíba, São José dos Campos, Brazil \\ ${ }^{3}$ Departamento de Ciências Farmacêuticas, Universidade Estadual de Londrina, Londrina, Brazil \\ ${ }^{4}$ Laboratório de Biotransformação, Universidade de Franca, Franca, Brazil \\ Email: janirafael@yahoo.com.br
}

Received June 18, 2013; revised July 18, 2013; accepted July 31, 2013

Copyright (c) 2013 Janice A. Rafael et al. This is an open access article distributed under the Creative Commons Attribution License, which permits unrestricted use, distribution, and reproduction in any medium, provided the original work is properly cited.

\begin{abstract}
Kaurenoic acid (KA), a kaurane-type diterpene extracted from leaves of Mikania hirsutissima, was previously reported as an inhibitor of vascular contractility mainly by blocking extracellular $\mathrm{Ca}^{2+}$ influx. The compound is known for several other biological activities such as antiparasitic, antispasmodic and antibacterial activity. The aim of the present study was to investigate the effect of KA on Aspergillus nidulans. KA $(0.3 \mathrm{mM})$ showed fungistatic activity against $A$. nidulans with visible hyphal elongation and morphology damage. These effects were reverted by $\mathrm{CaCl}_{2}$ addition showing that KA interferes with intracellular $\mathrm{Ca}^{2+}$ gradient in A. nidulans. This is the first report on the mechanism of action of KA involving calcium levels by altering the elongation of fungi hyphae.
\end{abstract}

Keywords: Aspergillus nidulans; Calcium Gradient; Fungistatic Activity; Kaurenoic Acid

\section{Introduction}

Many reports have shown that natural compounds obtained from plants have potential for the human treatment of diseases [1]. From these secondary metabolites, the kaurane-type diterpenes have been associated with several important biological properties, including antiparasitic effect [2], antispasmodic and relaxant actions on the smooth muscle [3,4], among others [5].

Members of this class of diterpenes have also been reported to have cytotoxic effects [6,7], antiproliferative action on tumour cell cultures [8], and significant antibacterial and antifungal activities, including effect against phytopathogenic fungi $[9,10]$.

According to Cotoras et al. [10], a kaurane-type diterpene, namely $3 \beta$-hydroxy-kaurenoic acid would produce changes in cell membrane permeability in the fungus $\mathrm{Bo}$ trytis cinerea with induced efflux of phosphorus, mainly by affecting membrane permeabilization, suggesting by the interaction of the diterpene with some membrane constituents. It is known that a tip-high cytoplasmic cal-

${ }^{*}$ Corresponding author. cium gradient is required for hyphal growth in most of fungi. The calcium concentration dependence of growth may relate directly to biochemical functions of calcium in hyphal extension, such as vesicle fusion and enzyme activation during cellular expansion [11]. Because KA was previously reported as an inhibitor of vascular contractility mainly by blocking extracellular $\mathrm{Ca}^{2+}$ influx [4] herein to learn more about the relation between kaurane diterpenes and fungi, a study was undertaken to analyze the effect of kaurenoic acid (Figure 1) on hyphal and growth in a fungus well characterized as Aspergillus nidulans. The effect of verapamil, a well known calcium channel blocker, was compared with KA.

\section{Materials and Methods}

\subsection{Strains and Growth Conditions}

Aspergillus nidulans strain FGSC A26 (biA1, veA1) was maintained according Vanzela and Said [12] and grown at $30^{\circ} \mathrm{C}$ for seven days before inoculating them on the culture medium (MM) containing nitrate salts and trace elements prepared according to Käfer [13] supplemented 


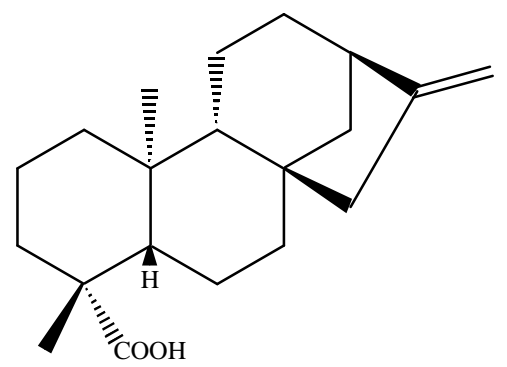

Figure 1. Chemical structure of KA (kaurenoic acid).

with $0.5 \%$ glucose and $0.02 \mu \mathrm{g} / \mathrm{mL}$ biotin; $1.8 \%$ agar was added and the $\mathrm{pH}$ was adjusted to 6.0.

\subsection{Isolation of Kaurenoic Acid (KA)}

Certified dried leaves of Mikania hirsutissima (1.0 kg) were purchased from "Nutri Comércio de Ervas LTDA", São Paulo, SP, Brazil. The plant material was pulverized and then exhaustively extracted with dichloromethane (3.5 L) at room temperature, to give $42.0 \mathrm{~g}$ of crude extract, which was suspended in $300 \mathrm{~mL}$ of methanol- $\mathrm{H}_{2} \mathrm{O}$ (9:1) and filtered. The soluble fraction was partitioned using $n$-hexane (300 mL, four times), resulting $6.0 \mathrm{~g}$ of hexane-soluble fraction after solvent evaporation under reduced pressure. The $n$-hexane-soluble fraction was chromatographed over Si gel 60 (0.063 - $0.200 \mathrm{~mm})$ using vaccum liquid chromatography [14] with $n$-hexane and increasing amounts of ethyl acetate as eluents $(250 \mathrm{~mL}$ each fraction). The second fraction (1730.0 mg) was washed with cold methanol, to afford pure KA (ent-kaur16(17)-en-19-oic acid; $800.0 \mathrm{mg}$ ), which was identified by spectroscopic analysis and comparison with literature data [15].

\subsection{Preparations of Extracts}

KA was dissolved in dimethylsulfoxide (DMSO) to make a $6.6 \mathrm{mM}$ stock solution and added to a final concentration of $0.3 \mathrm{mM}$. Verapamil $\left(\mathrm{Ca}^{2+}\right.$ channel blocker) was dissolved in DMSO to make $50 \mathrm{mM}$ stock solution and added to a final concentration of $1 \mathrm{mM}$. All aqueous solutions were sterilized by filtration. EGTA, a $\mathrm{Ca}^{2+}$ chelator, was dissolved in distilled water.

\subsection{Analysis of Hyphal Morphology}

For all cultures conidia were inoculated $\left(1 \times 10^{2}\right.$ conidia $)$ on dialysis membranes overlying $\mathrm{MM}$ in PETRI dishes and incubated at $30^{\circ} \mathrm{C} \pm 1^{\circ} \mathrm{C}$ for $30 \mathrm{~h}$ (pre-incubation time). Afterwards, all the chemicals were applied directly onto the cultures at $30^{\circ} \mathrm{C} \pm 1^{\circ} \mathrm{C}$ for 6,8 and $24 \mathrm{~h}$. The final concentration of solvents added to the cultures was adjust to less than 1 per cent and had no discernible effects on fungal growth. All treatments were performed at least two times with duplicates each. The hyphae were observed and photographed under an Olympus binocular stereomicroscope with transmitted light.

\section{Results}

Our results demonstrate that in presence of KA the hyphae lost the polarity and the addition of $\mathrm{CaCl}_{2}$ solution reverts this effect. Aspergillus nidulans showed alteration in the presence of $0.3 \mathrm{mM} \mathrm{KA}$ while at the same time 1 $\mathrm{mM}$ verapamil didn’t. KA affected hyphal morphology, inducing the lost of polarity after 6 and $8 \mathrm{~h}$ (Figures 2(D) and (E)). $\mathrm{CaCl}_{2}(500 \mathrm{mM})$ addition have reverted this morphological alteration (Figure 2(F)). However, abnormal hyphae and branching were decreased after $24 \mathrm{~h}$ (not shown).

\section{Discussion}

Plants have developed an arsenal of chemicals compounds in order to survive attacks by microbial invasion. Until now, many different antifungal compounds have been isolated from several plants. Since these compounds have relatively novel chemical structures and antifungal mechanisms, there has been a growing interest of antifungal compounds [16].

The establishment and maintenance of cell polarity in Aspergillus nidulans depend on the integration of many signaling networks including the calcium signaling and NDR (nuclear Dbf2-related) protein-kinase signaling pathway [17]. Many reports have shown also the importance of subfamily Rho of $\mathrm{G}$ proteins in governing the hyphal polarity establishment and maintenance. According to Kafer [18] in A. nidulans, microtubules and the Rho Cdc42 pathways regulate the formation of a stable axis of hyphal polarity. In $A$. niger RhoA GTPase is crucial for polarity establishment and polarity [19] and in mammalian the maintenance of arterial tone requires $\mathrm{Ca}^{2+}$ channel-dependent RhoA activation [20].

It is known that calcium is a vital intracellular second messenger involved in many other cellular functions as fungal growth, thus its influx and removal from cytoplasm have to be well regulated. In $A$. nidulans the levels of intracellular $\mathrm{Ca}^{2+}$ were higher in glucose than in pectin presence, demonstrating the $\mathrm{Ca}^{2+}$ importance when the fungi was growing fast [21]. The results presented here suggest that KA $(0.3 \mathrm{mM})$ altered the intracellular calcium homeostasis which by different mechanisms damaged the polarity and morphology of hyphae. The potential of verapamil and KA is apparently not identical. The voltage-dependent $\mathrm{Ca}^{2+}$ channel blocker Verapamil (1 $\mathrm{mM}$ ) promoted neither hiperbranching or abnormal hyphae nor lost polarity since 6 h until 24 h (Figures 2(G)(I)).

However, neither the identity of the internal store system nor the regulatory mechanisms controlling $\mathrm{Ca}^{2+}$ re- 

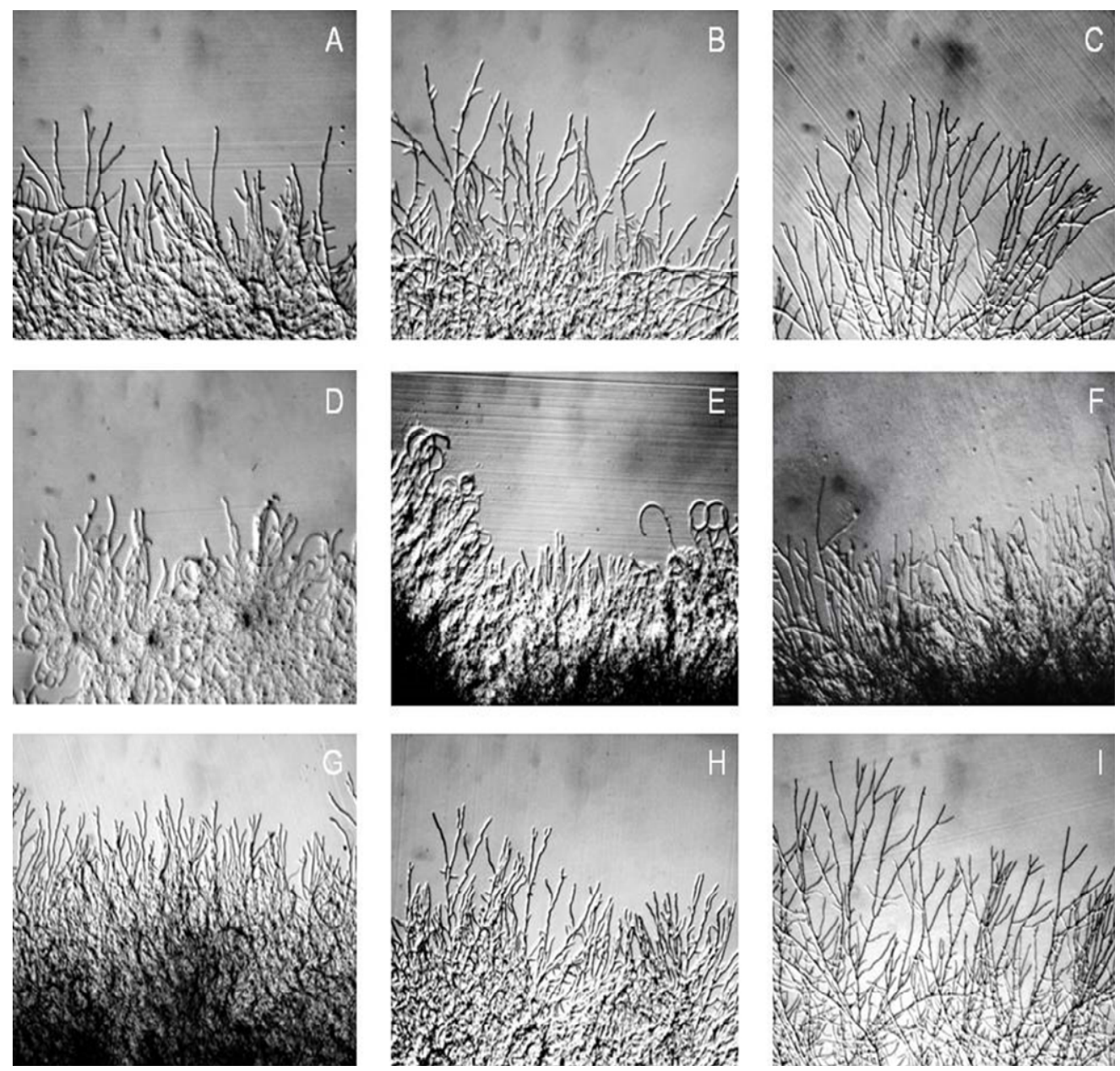

Figure 2. Effect of Kaurenoic acid and Verapamil in A. nidulans. Hyphae grown in medium supplemented with glucose $0.5 \%$ $(w / v)$ and in absence KA were analyzed after $6 \mathrm{~h}$ (A) $8 \mathrm{~h}$ (B) and $24 \mathrm{~h}$ (C). KA (0.3 mM) was added after $30 \mathrm{~h}$ of incubation in $(D)$ and $(E)$ and analized after $(6 \mathrm{~h})$ and $(8 \mathrm{~h})$ respectively. $\mathrm{CaCl}_{2}(500 \mathrm{mM})$ was added in cultures $(\mathrm{E})$ and analysed after $8 \mathrm{~h}$ (F). Verapamil (1 mM) was added and the cultures were analyzed after 6 (G), 8 (H) and 24 (I) hours. The preparations were viewed under stereomicroscope. Magnifications: $\times 56$.

leased from these stores are well known. In the present work our results indicated that KA reduced the hyphal polarity probably for altering the intracellular $\mathrm{Ca}^{2+}$ levels that consequently decreased the fungal growth. This is the first report on the mechanism of action of KA involving calcium levels altering the elongation of fungi hyphae, indicating that KA and its closely related analogues could be used as fungistatic agent.

\section{Acknowledgements}

J. A. R. received a Doctoral Fellowship from Coordenação de Apoio de Pessoal de Nível Superio (CAPES) and N. S. A. would like to thank Universidade Estadual de Londrina and Fundação Araucária.

\section{REFERENCES}

[1] J. D. Newman, "Natural Products as Leads to Potential
Drugs: An Old Process or the New Hope for Drug Discovery?” Journal of Medicinal Chemistry, Vol. 51, No. 9, 2001, pp. 2589-2599. doi:10.1021/jm0704090

[2] R. Batista, J. L. Humberto, E. Chiari and A. B. De Oliveira, "Synthesis and Trypanocidal Acitivy of ent-Kaurane Glycosides,” Bioorganic Medicinal Chemistry, Vol. 15, No. 1, 2007, pp. 541-544. doi:10.1016/j.bmc.2006.09.048

[3] S. R. Ambrosio, C. R. Tirapelli, F. B. Da Costa and A. M. De Oliveira, "Kaurane and Pimarane-Type Diterpenes from the Viguiera Species Inhibit Vascular Smooth Muscle Contractility," Life Sciences, Vol. 79, No. 10, 2006 , pp. 925-933. doi:10.1016/j.lfs.2006.05.015

[4] C. R. Tirapelli, S. R. Ambrósio, F. B. Da Costa, S. T. Coutinho, D. C. R. De Oliveira and A. M. De Oliveira, "Analysis of the Mechanisms Underlying the Vasorelaxant Action of Kaurenoic Acid in the Isolated Rat Aorta," European Journal of Pharmacology, Vol. 492, No. 2-3, 2004, pp. 233-241. doi:10.1016/j.ejphar.2004.04.003

[5] B. B. De Andrade, M. R. Moreira, S. R. Ambrósio, N. A. 
J. C. Furtado, W. R. Cunha, V. C. G. Heleno, N. A. Silva, M. R. Simão, E. M. P. Rocha, C. H. G. Martins and R. C. S. Veneziani, "Evaluation of Ent-Kaurenoic Acid Derivatives for Their Anticariogenic Activity," Natutral Products Communication, Vol. 6, No. 6, 2011, pp. 777-780.

[6] J. R. Hanson, “Diterpenoids," Natural Products Reports, Vol. 23, No. 6, 2006, pp. 875-885. doi:10.1039/b516326a

[7] E. L. Ghisalberti, "The Biological Activity of Naturally Occurring Kaurane Diterpenes," Fitoterapia, Vol. 68, No. 4, 1997, pp. 303-325.

[8] E. Mongelli, A. B. Pomilio, J. B. Sanchez, F. M. Guerra and G. M. Massanet, "Ent-Kaur-16-en-19-Oic Acid, a KB Cells Cytotoxic Diterpenoid from Elaeoselinum foetidum," Phytotherapy Research, Vol. 16, No. 4, 2002, pp. 387-388. doi:10.1002/ptr.955

[9] S. R. Ambrosio, N. A. J. C. Furtado, D. C. R. De Oliveira, F. B. Da Costa, C. H. G. Martins, T. C. De Carvalho and R. C. S. Veneziani, "Antimicrobial Activity of Kaurane Diterpenes against Oral Pathogens," Zeitschrift fur Naturforschung C, Vol. 63c, No. 5-6, 2008, pp. 326-330.

[10] M. Cotoras, C. Folch and L. Mendoza, "Characterization of the Antifungal Activity on Botrytis cinerea of the Natural Diterpenoids. Kaurenoic Acid and 3- $\beta$-HydroxiKaurenoic Acid," Journal of Agricultural and Food Chemistry, Vol. 52, No. 10, 2004, pp. 2821-2826. doi:10.1021/jf030672j

[11] L. B. Silverman-Gravila and R. R. Lew, "Calcium Gradient Dependence of Neurospora crassa hyphal Growth," Microbiology, Vol. 149, No. 9, 2003, pp. 2475-2485. doi:10.1099/mic.0.26302-0

[12] W. S. Sung and D. G. Lee, "In vitro Candidacidal Action of Korean Red Ginseng Saponins against Candida albicans," Biological and Pharmaceuthical Bulletin, Vol. 31, No. 1, 2008, pp. 139-142. doi:10.1248/bpb.31.139

[13] L. Gao, Y. Song, J. Cao, S. Wang, H. Wei, H. Jiang and L. $\mathrm{Lu}$, "Osmotic Stabilizer-Coupled Suppression of NDR Defectes Is Dependent on the Calcium-Calcineurin Signaling Cascade in Aspergillus nidulans," Cellular Signalling, Vol. 23, No. 11, 2011, pp. 1750-1757. doi:10.1016/j.cellsig.2011.06.009

[14] A. Virag, M. P. Lee, H. Si and S. D. Harris, "Regulation of Hyphal Morphogenesis by cdc42 and rac1 Homologues in Aspergillus Nidulans," Molecular Microbiology, Vol. 66, No. 6, 2007, pp. 579-596.

[15] M. J. Kwon, M. Arentshorst, E. D. Roos, C. A. Van den Hondel, V. Meyer and A. F. Ram, "Functional Characterization of Rho GTPases in Aspergillus niger Uncovers Conserved and Diverged Roles of Rho Proteins within Filamentous Fungi," Molecular Microbiology, Vol. 79, No. 5, 2011, pp. 1151-1167. doi:10.1111/j.1365-2958.2010.07524.x

[16] M. Fernández-Tenorio, C. Porras-González, A. Castellano, A. Del Valle-Rodríguez, J. López-Barneo and J. Ureña, "Metabotropic Regulation of RhoA/Rho-Associated Kinase by L-Type $\mathrm{Ca}^{2+}$ Channels: New Mechanism for Depolarization-Evoked Mammalian Arterial Contraction," Circulation Research, Vol. 108, No. 1, 2011, pp. 1348-1357. doi:10.1161/CIRCRESAHA.111.240127

[17] A. P. F. C. Vanzela and S. Said, "Evidence for Carbon Source Regulated Protein Kinase A and Protein Kinase C Signaling in the Duplication Cycle, Polarization and Septum Formation in Aspergillus nidulans," Microbiological Research, Vol. 157, No. 3, 2002, pp. 239-247. doi:10.1078/0944-5013-00156

[18] E. Käfer, "Meiotic and Mitotic Recombination in Aspergillus and Its Chromosomal Aberrations," Advances in Genetics, Vol. 19, 1977, pp. 33-131. doi:10.1016/S0065-2660(08)60245-X

[19] S. W. Pelletier, H. P. Chokshi and H. K. Desai, "Separation of Diterpenoid Alkaloid Mixtures Using Vacuum Liquid Chromatography," Journal of Natural Products, Vol. 49, No. 5, 1986, pp. 892-900. doi:10.1021/np50047a021

[20] A. M. Do Nascimento and D. C. R. De Oliveira, "Kaurene Diterpenes and Other Chemical Constituents from Mikania stipulacea (M. Vahl) Willd.," Journal of the Brazilian Chemical Society. Vol. 12, No. 4, 2001, pp. 552-555. doi:10.1590/S0103-50532001000400019

[21] J. A. Rafael and S. Said, "The Difference in Calcium Levels in Aspegillus nidulans Grown on Glucose or Pectin,” Advances in Microbiology, Vol. 2, 2012, pp. 117121. 